\title{
Resistencia a la terapia antirretroviral en pacientes infectados con el virus VIH-1 en Chile 2002-2005
}

\author{
Alejandro Afani $S^{1}$, Laura 0 rellana $\mathbf{R}^{1}$, Paula Duarte $\mathrm{J}^{\mathbf{1}}$, \\ Wiliam Acevedo $\mathbf{M}^{2 a}$, 0 mar Morales $B^{3 b}$, Marcelo Wolff $\mathbf{R}^{3}$, \\ Patricia Vásquez ${ }^{3}$, Carlos Beltrán ${ }^{3}$. \\ Resistance to anti-retroviral therapy \\ in Chilean patients with HIV-1 \\ from 2002 to 2005
}

Background: Resistance limits the effectiveness of anti-retroviral therapy. In Chile, there is free access to highly active anti-retroviral therapy since 2001, but there is no information about the frequency of mutations associated to drug resistance. Aim: To determine the most common mutations associated to anti-retroviral drug resistance in Chile. Materials and Methods: Retrospective study of 710 genotype analysis coming from 568 patients aged 22 to 70 years (85\% males) with virological failure. The analysis was performed using a commercially available sequencing kit (Trugene HIV-1 genotypic assay from Bayer S.A). Results: Mean CD4+ cell count and viral load were 154 cells/ $\mu$ l and 228784 RNA copies/ml, respectively. The frequency of resistance to nucleoside RT inhibitors (NRTI), non nucleoside RT inhibitors (NNRTI) and protease inhibitors (PI) was $71 \%, 62 \%$ and $22 \%$, respectively. The most common mutations found were T215Y (46\%), L10F (44\%), M184V (38\%), K103N (35\%) and M41L (32\%). Fifty five percent of mutations corresponded to the TAM (thymidine analogue mutations) group. Multiresistance was $47 \%$ to NNRTI, $7 \%$ to NRTI, $4 \%$ to PI and $0.7 \%$ to all groups. During the four years of the study, there was a significant increase in NNRTI resistance. Conclusions: These data provides important information about the epidemiology of drug resistance mutations and should help to design new HAART strategies (Rev Méd Chile 2007; 135: 1237-44).

(Key words: Anti-retroviral agents; HIV-1; Nucleosides).

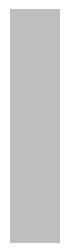

Recibido el 26 de octubre, 2006. Aceptado el 8 de mayo, 2007.

${ }^{1}$ Sección de Inmunología, Departamento de Medicina, Hospital Clínico de la Universidad de Chile, Santiago, Chile. ${ }^{2}$ Laboratorio de Medicina Molecular, Sección de Inmunología, Hospital Clínico de la Universidad de Chile, Santiago, Chile. ${ }^{3}$ Grupo ChiaC, Cohorte Chilena de SIDA.

aBioquímico

bAsesor Informático

Correspondencia a: Dr. Alejandro Afani S. Independencia 1027, Independencia, Santiago. Fax: (02) 7371991. E mail: aafani@vtr.net 
$\mathrm{E}^{\mathrm{s}}$ la última comunicación del Programa conjunto de las Naciones Unidas sobre el VIH/ SIDA, ONUSIDA, de diciembre de 2006, aproximadamente 39,5 millones de personas se encuentran infectadas por el virus de la inmunodeficiencia humana (VIH) en el mundo, y 2,9 millones han fallecido a causa de la epidemia. En Chile, actualmente se estima que habría 15 mil personas viviendo con el $\mathrm{VIH}, 85 \%$ de las cuales pertenecen al grupo de 20 a 49 años, con una relación hombre:mujer de 4:1, siendo la vía sexual la principal forma de transmisión en $94 \%$ de los casos $^{1,2}$.

La introducción de la terapia antirretroviral (TARV), ha disminuido la morbimortalidad de la infección por VIH, reduciendo la carga viral a niveles indetectables y restaurando el sistema inmune, evidenciado por el aumento del recuento de linfocitos TCD4 $(+)^{3-6}$. En Chile, el inicio de la TARV comenzó el año 1993 con monoterapia y posteriormente con biterapia hasta el año 1999. A partir de 2001 existe acceso ampliado a la TARV (triterapia), alcanzando una cobertura de $100 \%$ el año $2003^{2}$. Según datos aportados por la Cohorte Chilena de SIDA, actualmente 6.658 pacientes se encuentran en TARV. Aproximadamente $60,3 \%$ de los pacientes en TARV actual y en falla virológica, recibieron mono o biterapia, mientras que 19,3\% era virgen a TARV. Uno de los principales problemas asociados a la TARV es la falla virológica, debido a la aparición de resistencia (R) a las drogas antirretrovirales (ARV) 3,7 .

La resistencia a ARV es ocasionada por la aparición de mutaciones a nivel del gen pol, principalmente a nivel de la transcriptasa reversa y de la proteasa viral5,8. Dentro de las más importantes se encuentran las mutaciones TAM (thymidine associated mutations), seleccionadas por zidovudina (AZT) y estavudina (d4T). Estas mutaciones pueden generar resistencia cruzada a la mayońa de los inhibidores de la transcriptasa reversa (ITRN) no análogos de nucleósidos ${ }^{9-11}$. Las mutaciones TAM son M41L, D67N, K70R, L210W, T215Y y K219Q. De éstas, las que se asocian a un mayor grado de R son M41L, L210W y T215Y, denominadas TAM 1, en tanto la D67N, K70R y K219Q confieren menor grado de R, y se denominan TAM $2^{12,13}$. Las mutaciones M184V, K65R y L74V se asocian principalmente a $\mathrm{R}$ a lamivudina (3TC), tenofovir (TDF) y didanosina (DDI), respectivamente, sin embargo, pueden tam- bién conferir menor grado de $\mathrm{R}$ a otros ITRN ${ }^{7,12-15}$. La multimesistencia ( $\mathrm{R}$ a todas las drogas del grupo) en el grupo ITRN se puede dar por acumulación de mutaciones TAM, por el complejo Q151M, o por la doble inserción del codón 6913,16-18.

En el grupo de los inhibidores de nucleósidos no análogos de la transcriptasa reversa (ITRNN), la mutación K103N, seleccionada por efavirenz (EFV), al igual que la mutación Y188L seleccionada por nevirapina (NVP), confieren R cruzada a todo el grupo $7,10,12,13$.

La multirresistencia en este grupo se da por la sola presencia de una de estas mutaciones o la V106M, o bien por la acumulación de otras como: L100I, V106A, Y181C, G190S y M230L ${ }^{12,13}$.

En el grupo de los inhibidores de proteasa (IP) se presentan mutaciones específicas a algunas drogas como D30N, I50L, I50V y G48V, que confieren R a nelfinavir, atazanavir, fosamprenavir y saquinavir, respectivamente ${ }^{9,12,13}$. En general, el grupo de IP presenta una barrera genética más alta, es decir la $\mathrm{R}$ se presenta por la acumulación de varias mutaciones ${ }^{10,19,20}$. Las principales mutaciones asociadas a $\mathrm{R}$ en este grupo son: M46I, I54V, V82A, I84V y L90M, pero además, las mutaciones en los codones 10, 20, 32, 36, 63 y 71 entre otros, contribuyen en otorgar $\mathrm{R}$ en menor grado ${ }^{12,13}$.

El test de resistencia genotípica permite identificar la aparición de mutaciones asociadas a $\mathrm{R}$ en pacientes con falla virológica sometidos a TARV, permitiendo optimizar nuevos esquemas de tratamiento $7,18,22$. En Chile, el test de genotipificación se realiza desde el año 2002, su principal indicación en la actualidad es después de presentarse la primera falla virológica. No existen datos publicados acerca de la epidemiología de la resistencia en pacientes sometidos a TARV en la cohorte chilena ${ }^{2,8}$.

El objetivo de este trabajo es describir el tipo de mutaciones, su frecuencia y la forma en que ha evolucionado el perfil de resistencia a ITRN, ITRNN e IP en pacientes chilenos sometidos a TARV en cuatro años de estudio.

\section{Metodología}

Se realizó un estudio descriptivo retrospectivo de 710 estudios de genotipificación, correspondientes a 568 pacientes con infección por VIH-1 provenientes de todos los centros de atención de 
VIH/SIDA de Chile, los cuales fueron realizados durante los años 2002 a 2005. Todos los pacientes se encontraban en terapia antirretroviral altamente efectiva y tenían indicación de estudio de genotipificación por falla virológica, es decir, carga viral mayor a 1.000 copias/ml después de 6 meses de TARV efectiva o después de haber sido indetectable (menos de 80 copias/ml) ${ }^{24}$. Algunos pacientes tenían más de un estudio de genotipificación. El análisis se realizó mediante el ensayo genotípico TRUGENE $^{\text {TM }}$ HIV-1 (Bayer SA) ${ }^{21}$, disponible en el Laboratorio de Medicina Molecular del Hospital Clínico de la Universidad de Chile. Este ensayo se basa en la amplificación genética mediante reacción de polimerasa en cadena (PCR), para luego analizar la secuencia nucleotídica, identificando mutaciones en los genes de las enzimas transcriptasa reversa y proteasa virales implicadas en el desarrollo de resistencia ${ }^{21,22}$. Las características demográficas, etapa clínica, recuento de linfocitos TCD4+ y carga viral de los pacientes, fueron obtenidas de la base de datos de la Cohorte Nacional de SIDA del Grupo SIDA Chile. El análisis estadístico se realizó a través del programa SPSS Windstata versión 13 y Statcalc versión 4.

\section{RESULTADOS}

I) Características de los pacientes. Se analizaron 710 estudios de genotipificación, realizados durante los años 2002 a 2005. La edad promedio fue de 41

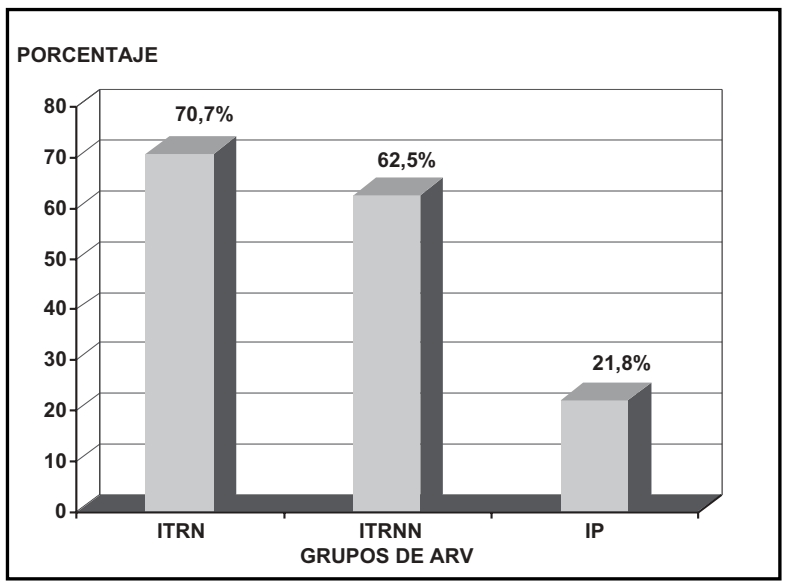

Figura 1. Prevalencia de resistencia por grupo de antirretrovirales.
Tabla 1. C aracterísticas generales de los pacientes

\begin{tabular}{|ll|}
\hline Edad & $\mathrm{n}: 568$ \\
Promedio: & 41 años (22-70 años) \\
Mediana: & 40 años \\
Género & \\
Masculino: & $85 \%$ \\
Femenino: & $15 \%$ \\
Clasificación CDC & \\
A1-A2 & $5,75 \%$ \\
B1-B2 & $5,75 \%$ \\
A3-B3-C & $88,4 \%$ \\
Recuento de CD4+ & \\
Promedio & $154 \mathrm{cel} / \mathrm{ml}(0-922)$ \\
Mediana & $121 \mathrm{cel} / \mathrm{ml}$ \\
Carga Viral & \\
Promedio & $228.784 \mathrm{copias} / \mathrm{ml}$ \\
Rango & $5.100-4.200 .000$ \\
Mediana & $74.000 \mathrm{copias} / \mathrm{ml}$ \\
\hline
\end{tabular}

años (22-70), 85\% fue de sexo masculino. El 88,4\% se encontraba en etapa clínica SIDA (A3-B3 y C) de la clasificación CDC de 1992. El recuento promedio de linfocitos TCD4+ fue de $154 \mathrm{cel} / \mathrm{ml}$ (0-922), y la carga viral promedio fue de 228.784 copias de RNA $\mathrm{viral} / \mathrm{ml}$ (5.100-4.200.000) (Tabla 1).

II) Análisis de resistencia. En el grupo de los ITRN, $70,7 \%$ presentó resistencia a por lo menos una droga del grupo, 62,5\% en el grupo ITRNN y de 21,8\% en el grupo de IP (Figura 1).

Las mutaciones individuales en los genes de la transcriptasa reversa y proteasa virales más frecuentemente encontradas fueron: T215Y (45,9\%), L10F (44,2\%), M184V (38,3\%), K103N (35,1\%) y M41L (32,4\%) (Figura 2). La resistencia de cada droga por grupo de antirretrovirales mostró una prevalencia similar en el grupo de los ITRN sobre 40\%, como se aprecia en la Figura 3. Destaca la menor resistencia de tenofovir, la cual fue de $21,4 \%$. La resistencia más alta en el grupo ITRNN fue nevirapina con $62,3 \%$, en tanto la resistencia a efavirenz fue de $48,9 \%$ (Figura 3). La resistencia en el grupo IP se mantiene baja, la mayor resistencia la presentó nelfinavir con $21,7 \%$. Destaca la menor prevalencia de resistencia con el uso de IP reforzado, lopinavir/ritonavir que fue de $6,1 \%(p<0,001)$ (Figura 4$)$. 


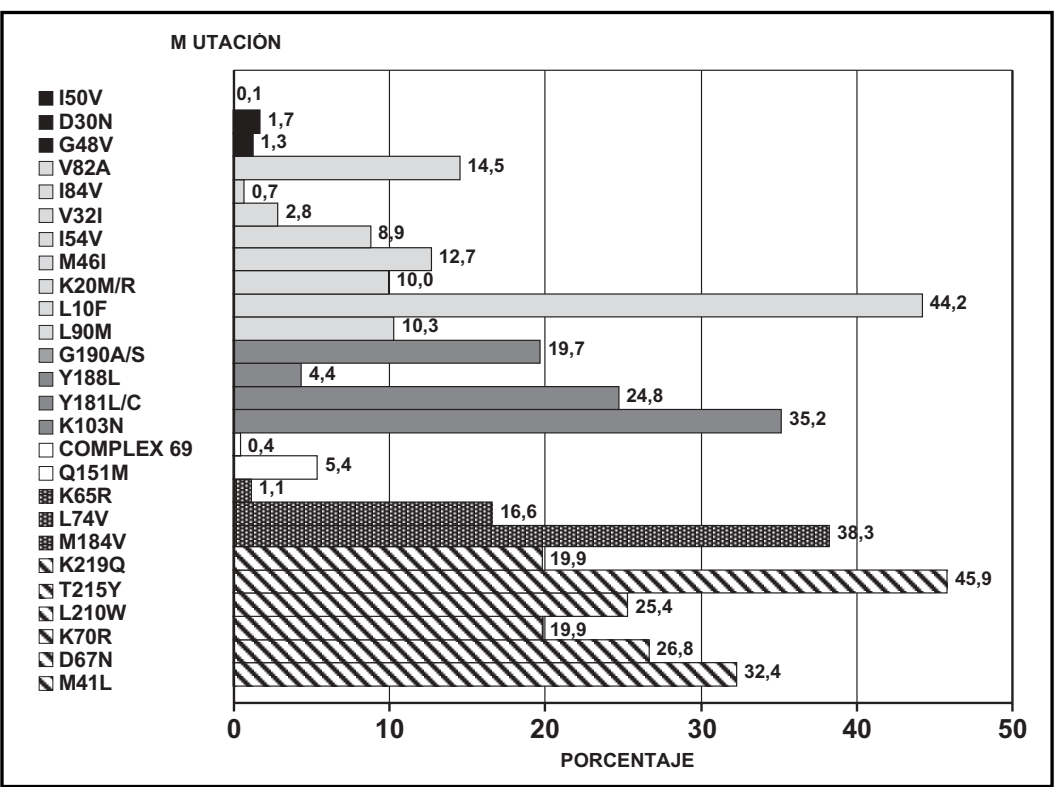

Figura 2. Prevalencia de mutaciones en los genes de la Transcriptasa reversa y Proteasa del virus VIH-1.

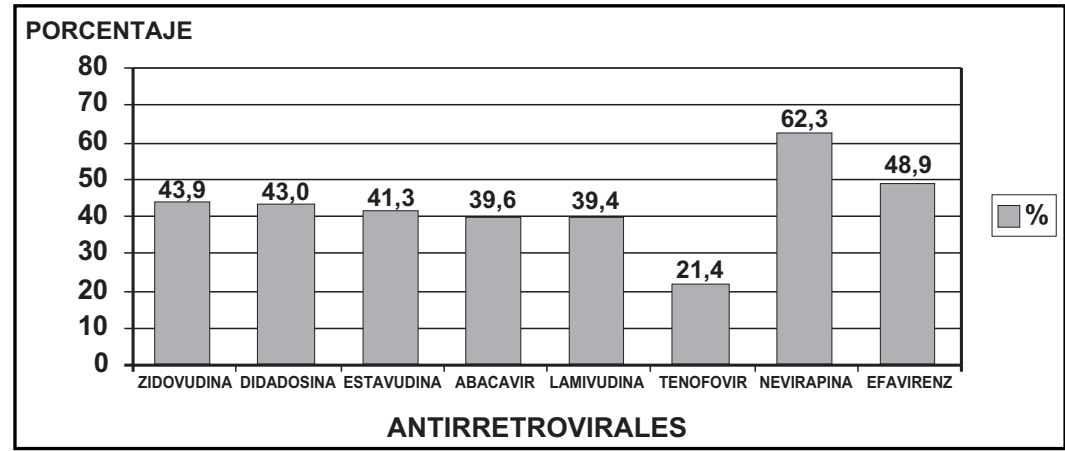

Figura 3. Prevalencia de resistencia a cada droga en el grupo ITRN e ITRNN

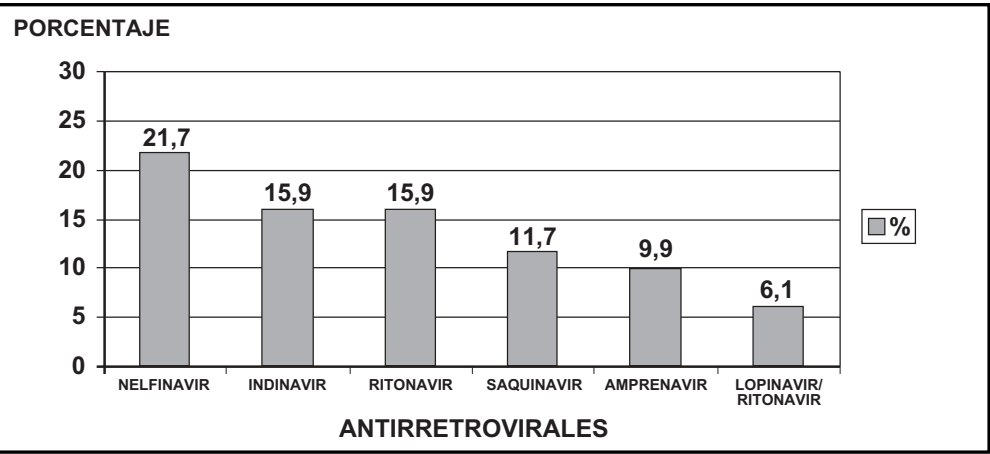

Figura 4. Prevalencia de resistencia a cada droga en el grupo IP 
El análisis de resistencia por grupo de mutaciones mostró que $54,5 \%$ presenta al menos una mutación TAM, 46,5\% presentó mutaciones específicas del grupo ITRN (M184V, L74V, K65R). Sólo 6,5\% presentó mutaciones que confieren multirresistencia a los ITRN, dada principalmente por la acumulación de mutaciones TAM, la aparición de mutaciones del complejo Q151 y el complejo de inserción del codón 69. El 39,6\% presentó mutaciones que confieren multimesistencia al grupo ITRNN. La resistencia específica a IP (G48V, D30N, I50V/L) fue sólo de 3\%, y la $\mathrm{R}$ al grupo IP fue de 1,87\%, debido principalmente a la acumulación de 60 más mutaciones.

En cuanto a la distribución de mutaciones TAM, 45,6\% presentó al menos una mutación del tipo TAM 1 (M41L, L210W, T215Y) y 32,8\% del grupo TAM 2 (K70R, D67N, K219Q).
El porcentaje de $\mathrm{R}$ a diferentes asociaciones de ITRN mostró la menor frecuencia de resistencia en las asociaciones AZT+3TC (15,6\%), D4T+3TC $(14,4 \%)$ y TDF+3TC $(8,6 \%)$ (Figura 5$)$.

La prevalencia de multimesistencia a cada grupo de antimetrovirales fue de $47,2 \%$ para el grupo ITRNN, 6,5\% para el grupo ITRN, 4,2\% para el grupo IP, y de $0,7 \%$ para las tres familias de ARV (Figura 6).

En cuanto a la evolución de la $\mathrm{R}$ a los diferentes grupos de drogas antirretrovirales, no se observaron diferencias significativas a lo largo de los 4 años de estudio. En el grupo ITRN se observa una prevalencia mantenida cercana a $70 \%$. El grupo ITRNN muestra una tendencia estadísticamente significativa ( $p<0,001)$ al aumento de la resistencia a partir del año 2003, con progresivo incremento hasta el año 2005. El grupo IP, muestra una

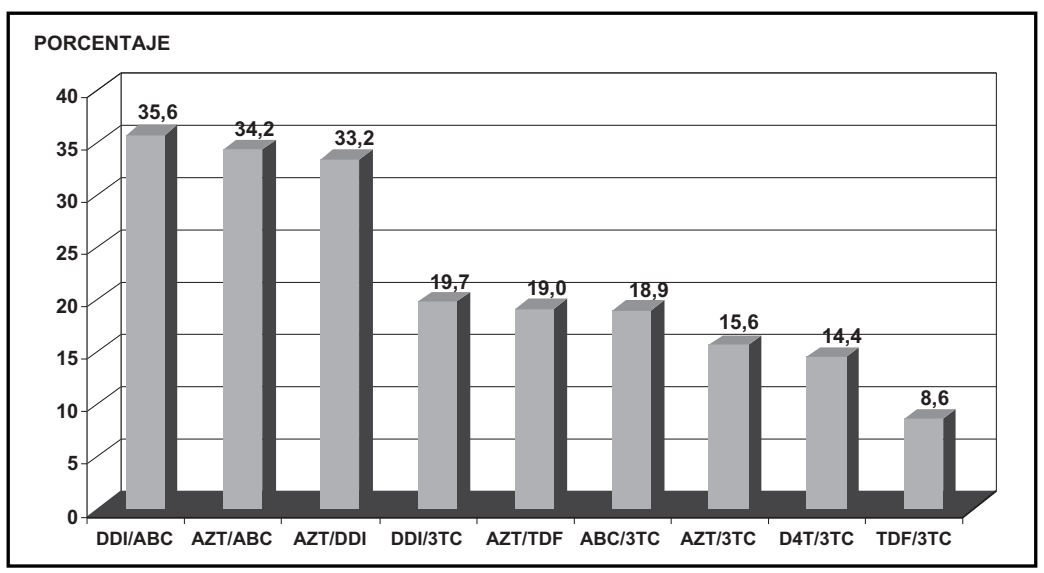

Figura 5. Prevalencia de resistencia a asociaciones de ITRN.

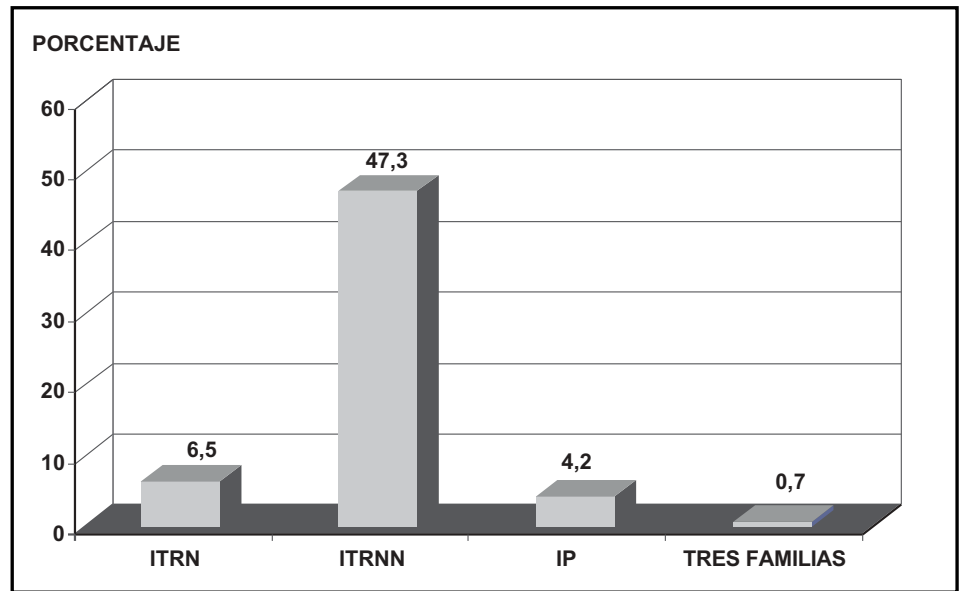

Figura 6. Frecuencia de multirresistencia por grupo de antirretrovirales y a las tres familias de antirretrovirales. 
tendencia a la disminución que no es estadísticamente significativa ( $p<0,423$ ) (Figura 7).

\section{DisCUSIÓN}

Este primer estudio de resistencia a la terapia antirretroviral en pacientes con infección VIH-1 en Chile, muestra resultados similares a los de estudios internacionales ${ }^{23}$. Este estudio ha permitido definir el perfil de mutaciones asociadas a $\mathrm{R}$ en Chile, reflejando que en pacientes en falla virológica, el mayor grado de $\mathrm{R}$ se observa en los grupos de los ITRN e ITRNN. Esto se debe principalmente al mayor uso de ITRN como primer esquema de tratamiento. Sin embargo, se observa un aumento de la $\mathrm{R}$ a los ITRNN a partir del año 2003, lo que se explica por el aumento del uso de este tipo de antirretrovirales en reemplazo de los IP como primera línea de tratamiento ${ }^{24}$.

En el caso de los ITRN, la mayoría de las drogas presenta un perfil de $\mathrm{R}$ cercano a $40 \%$, donde la zidovudina (AZT), con $43,9 \%$, es la droga que presenta más $\mathrm{R}$ de este grupo, lo que es esperable siendo la droga más ampliamente usada en los esquemas actuales de inicio de tratamiento, así como también fue utilizada anteriormente en nuestro país como monoterapia y en biterapia hace más de 10 años ${ }^{2,24}$.
El análisis de las mutaciones mostró una mayor prevalencia de mutaciones TAM, especialmente del tipo TAM 1, las cuales confieren mayor $\mathrm{R}$ a los ITRN, como son M41L, L210W y T215Y. Sin embargo, a pesar de la mayor prevalencia de mutaciones TAM, la mutación K65R se presentó en un muy bajo porcentaje $(1,13 \%)$ por lo que destaca tenofovir como droga alternativa dentro del grupo de los análogos de nucleósidos ${ }^{14}$. Pese a que en nuestro país la $\mathrm{R}$ a tenofovir $(21,4 \%)$ está dada principalmente por la acumulación de 3 o más mutaciones TAM, incluyendo la M41L o L210W, es la droga con menor R dentro del grupo ITRN.

En el grupo ITRNN, nevirapina es la droga con mayor R (62,3\%). Además, debido a la baja barrera genética de los ITRNN, la aparición de una mutación asociada a $\mathrm{R}$ de una droga, puede conferir resistencia al grupo completo, como es el caso de la sola mutación K103N o Y188L que otorgan resistencia tanto a efavirenz como nevirapina.

La resistencia a los IP se mantiene baja debido a su uso actual como droga de segunda línea en esquemas de rescate, además, los IP presentan una mayor barrera genética, lo que implica la acumulación de varias mutaciones para generar resistencia ${ }^{9}$. Destaca nelfinavir $(21,7 \%)$ como la droga con mayor R dentro de este grupo, sin embargo no ha

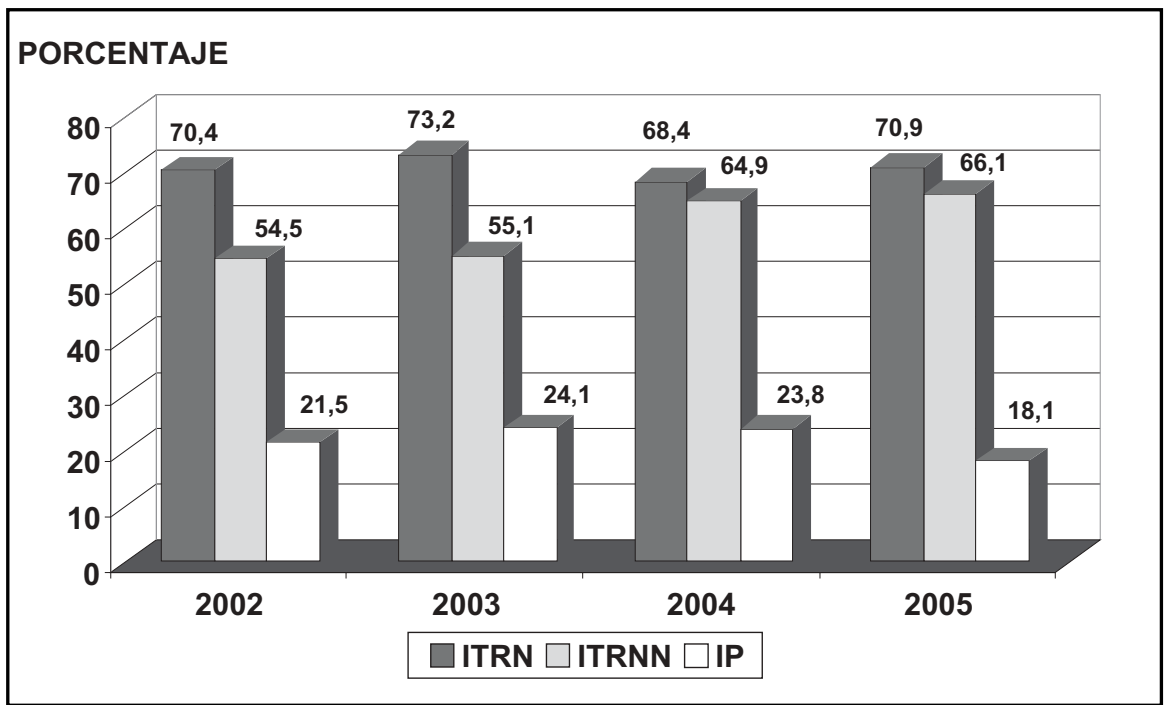

Figura 7. Evolución de la resistencia por grupo de antirretrovirales durante los años 2002 a 2005. 
sido la más usada de los IP. Esto es debido a que la $\mathrm{R}$ se puede presentar tanto por acumulación de mutaciones, como por la sola presencia de la mutación D30N. En el grupo IP, lopinavir/ritonavir $(6,1 \%)$ es la droga con menor $R$, debido a que ésta requiere mayor acumulación de mutaciones primarias y secundarias.

Dentro de las asociaciones de análogos de nucleósidos, las asociaciones de AZT+3TC, D4T+3TC, y TDF+3TC son las que presentaron menor porcentaje de $\mathrm{R}$, validando su uso como parte del primer esquema de tratamiento, e incluso en pacientes con falla virológica ${ }^{16}$.

Con respecto a la aparición de multirresistencia por grupo de antirretrovirales, se observa un bajo porcentaje en el grupo ITRN (6,5\%). Esto puede explicarse por el bajo porcentaje de las mutaciones de multirresistencia como el complejo Q151, y la inserción del codón 69, cuya prevalencia en gene$\mathrm{ral}$ es menor a $5 \% 21$. La multirresistencia en el grupo ITRNN $(47,3 \%)$ se ha incrementado sobre todo por el aumento de la mutación K103N ${ }^{17,20}$. En el grupo de los IP, la multimesistencia $(4,2 \%)$ sigue siendo baja en la actualidad, y es de gran relevancia que en Chile la multirresistencia a las tres familias de drogas sea de $0,7 \%$, lo que permite disponer aún de armas terapéuticas eficientes en pacientes en falla virológica.

\section{REFERENCIAS}

1. Onusida. Situación mundial de la epidemia de SIDA: Diciembre de 2006. http//:www.unaids.org/ en/HIVdata/epi2006. Consultado en enero 2007.

2. Minsal. Situación epidemiológica del VIH/SIDA en Chile 1984-2004. Julio 2006. En http:// epi.minsal.cl/epi/html/bolets/reportes/SitVIHSIDA2004. Consultado en enero de 2007.

3. Mocroft A, Velia S, Benfield TL, Chiesi A, Miler V, Gargalianos $P$ Et al. Changing patterns of mortality across Europe in patients infected with HIV-1. EuroSIDA Study Group. Lancet 1998; 352: 1725-30.

4. Paleila Jr FJ, Delaney KM, Moorman AC, Loveless MO, FunRER J, SATTEN GA et AL. Declining morbility and mortality among patients with advances human immnunodeficiency virus infection. HIV
En cuanto a la evolución de la $\mathrm{R}$ a lo largo de los 4 años de seguimiento, no se observaron cambios significativos en el perfil evolutivo de $\mathrm{R}$. Cabe destacar una tendencia al aumento de la resistencia a los ITRNN en los últimos 2 años, y la disminución en la resistencia del grupo IP durante el último año, debido a su menor uso actual como primer esquema, siendo utilizadas como drogas de rescate, en pacientes con falla virológica a la TARV $^{9}$.

Finalmente, este estudio ha permitido conocer el perfil de $\mathrm{R}$ a la terapia antirretroviral en pacientes con fallo virológico en Chile, identificando las mutaciones más frecuentes y su impacto en la aparición de R. Estos resultados han permitido optimizar la TARV, y han contribuido al desarrollo de nuevas guías clínicas de tratamiento de pacientes con infección VIH/SIDA en nuestro país ${ }^{24}$. Será de gran relevancia continuar este estudio, con el fin de conocer las tendencias en relación a $\mathrm{R}$ a la TARV en el tiempo, como también será importante conocer el perfil actual de resistencia en pacientes vírgenes a TARV ${ }^{8}$, ya que al igual que en países desarrollados, este análisis podría determinar en Chile la necesidad de realizar el estudio de resistencia previo a TARV $^{25}$.

Outpatients Study Group. N Engl J Med 1998; 338: 853-60.

5. Vittinghoff E, Scheer S, O'Mauey P, Colfax G, HolmBerg SD, BuCHBindER SP. Combination antiretroviral therapy and recent declines in AIDS incidente and mortality. J Infect Dis 1999; 179: 717-20.

6. Chew C, Potter S, Wang B, Min Wang Y, Shaw C, DwYer D, SAKSENA N. Assessment of drug resistance mutations in plasma and peripheral blood mononuclear cells at different plasma viral loads in patients receiving HAART. J Clin Virol 2005; 33: 206-16.

7. Ciavel F, Hance A. HIV Drug Resistance. N Engl J Med 2004; 350: 1023-35.

8. Afani A, Ayala M, Meyer A, Cabrera R, Acevedo W. Resistencia primaria a terapia antirretroviral en pacientes con infección por VIH/SIDA en Chile. Rev Méd Chile 2005; 133: 295-301. 
9. DeEKS S. Treatment of antiretroviral-drug-resistant HIV-1 infection. The Lancet 2003; 362: 2000-11.

10. MENÉNDEZ-ARIAS L. Targeting HIV: antiretroviral therapy and development of drug resistance. Trends Pharmacol Sci 2002; 23: 381-8.

11. Sarafianos S, Hughes S, Arnold E. Designing antiAIDS drugs targeting the major mechanism of HIV-1 RT resistance to nucleoside analog drugs. Int J Biochem Cell Biol 2004; 36: 1706-15.

12. GaLint J. Antiretroviral Drug Resistance and Resistance Testing. Internacional AIDS SocietyUSA. Top HIV Med 2005; 13: 138-42.

13. Johnson V, Brun-Vézinet F, Clotet B, Conway B, Kuritzkes D, Pillay D et al. Update of the Drug Resistance Mutations in HIV-1: Fall 2006. Internacional AIDS Society-USA. Top HIV Med 2006; 14: 125-30.

14. MiLeR M. K65R, TAMs and Tenofovir. AIDS Reviews 2004; 6: 22-33.

15. WAINBERG M. The impact of the M184V substitution on drug resistance and viral fitness. Expert Rev Anti Infect Ther 2004; 2: 147-51.

16. Rezende L, PRASAd V. Nucleoside-analog resistance mutations in HIV-1 reverse transcriptase and their influence on polymerase fidelity and viral mutation rates. Int J of Biochem Cell Biol 2004; 36: 1716-34.

17. IVERSEN AK, SHAFER RW, WeHRLY K. Multidrugresistant human immunodeficiency virus type 1 strains resulting from combination antiretroviral therapy. J Virol 1996; 70: 1086-90.
18. Hoffman C, Kamps S. HIV drug resistance. HIV Medicine 2006.http://www.hivmedicine.com. Consultado octubre 2006.

19. Hertogs K, Bloor S, Kemp SD. Phenotypic and genotypic analysis of clinical HIV-1 isolates reveals extensive protease inhibitor cross-resistance a survey of over 6000 samples. AIDS 2000; 14: 1203-10.

20. TANg J, PIшaY D. Transmission of HIV-1 drug resistance. J Virol 2004; 30: 1-10.

21. Kuritzkes DR, Grant RM, Feorino P, Griswold W, Hoover M, Young R et al. Performance characteristics of the TRUGENE HIV-1 Genotyping Kit and the Opengene DNA Sequencing System. J Clin Microbiol 2003; 41: 1594-9.

22. García-Lerma J, Heneine W. Resistance of Human Immunodeficiency Virus Type 1 to Reverse Transcriptase and Protease Inhibitors: Genotypic and Phenotypic Testing. J Clin Virol 2001; 21: 197-212.

23. Tanura A, Caridea E, Dantas MC, Morgado MG, MeLo DL, Borges S et AL. Prevalence of mutations related to HIV-1 antiretroviral resistance in Brazilian patients failing HAART. J Clin Virol 2002; 25: 39-46.

24. Conasida. Chile. Guía clínica para la atención de las personas adultas que viven con VIH/SIDA. 2005.

25. Wensing AM, Van De Vijver DA, Angarano G. Prevalence of drug-resistant HIV-1 variants in untreated individuals in Europe: implications for clinical management. J Infect Dis 2005; 192: 958-66. 